\title{
Eli FRANCo, (éd.), Dagmar EINER, (collab.), Yogic Perception, Meditation and Altered States of Consciousness
}

Vienne, Verlag der Österreichischen Akademie der Wissenschaften, 2009, $483 \mathrm{p}$.

\section{André Padoux}

\section{CpenEdition} Journals

Édition électronique

URL : http://journals.openedition.org/assr/22590

DOI : $10.4000 /$ assr.22590

ISSN : $1777-5825$

Éditeur

Éditions de l'EHESS

Édition imprimée

Date de publication : 31 décembre 2010

Pagination : 9-242

ISBN : 9782713223013

ISSN : 0335-5985

Référence électronique

André Padoux, «Eli franco, (éd.), Dagmar einer, (collab.), Yogic Perception, Meditation and Altered States of Consciousness ", Archives de sciences sociales des religions [En ligne], 152 | octobredécembre 2010, document 152-53, mis en ligne le 12 mai 2011, consulté le 21 septembre 2020. URL : http://journals.openedition.org/assr/22590 ; DOI : https://doi.org/10.4000/assr.22590

Ce document a été généré automatiquement le 21 septembre 2020.

(C) Archives de sciences sociales des religions 


\section{Eli FRANCO, (éd.), Dagmar EINER, (collab.), Yogic Perception, Meditation and Altered States of Consciousness}

Vienne, Verlag der Österreichischen Akademie der Wissenschaften, 2009, $483 \mathrm{p}$.

André Padoux

\section{RÉFÉRENCE}

Eli FRANCO, (éd.), Dagmar EINER, (collab.), Yogic Perception, Meditation and Altered States of Consciousness, Vienne, Verlag der Österreichischen Akademie der Wissenschaften, 2009, $483 \mathrm{p}$.

1 Ce gros volume rassemble les exposés faits à Vienne, en juin 2006, au cours d'une conférence organisée par l'Académie des Sciences d'Autriche dans le cadre d'un projet de recherche, poursuivi de 2002 à 2004, sur la notion de perception para- et supranormale dans la tradition épistémologique bouddhique. Ce projet est lui-même inclus dans un ensemble de recherches plus vaste, "The epistemological-logical tradition in India and Tibet», que dirigea pendant plus de vingt ans l'éminent tibétologue Ernst Steinkellner. Comme le note Eli Franco dès le début de son importante introduction, l'idée que la méditation puisse être la source de perceptions extrasensorielles, d'une forme de connaissance particulière, est présente en Inde dès les temps les plus anciens, cette méditation tendant très tôt à être liée au renoncement et à l'ascèse. Cette attitude est celle des śramana, des ascètes renonçants, que représentent notamment le jinisme et le bouddhisme, mais elle s'est également affirmée du côté brahmanique/hindou, associée dans les deux cas à l'idée que le monde où vivent les êtres humains est empli de douleur et qu'il convient de s'en libérer par le 
renoncement, la méditation et l'ascèse. Ces thèses sont notamment celles du Yoga dont la forme dite "classique», celle des Yogasūtra de Patañjali, a d'ailleurs été fortement influencée par le bouddhisme, qui, à son tour, en est venu à l'inclure dans ses conceptions et ses pratiques. C'est de cet aspect bouddhique que traite pour l'essentiel ce volume.

2 La plupart de ces mouvements ascétiques ont en commun l'idée que l'on parvient à la libération par une connaissance intuitive de la nature réelle du monde, une perception extrasensorielle donneuse d'omniscience, que cette vision de la réalité soit posée comme innée, propre au plan le plus haut de la conscience, ou comme acquise par une ascèse purificatrice, la conscience étant toutefois, dans les deux cas, censée avoir une capacité latente de perception "paranormale», aptitude qui doit toutefois être actualisée par la méditation (jhāna en pali, dhyāna, en sanskrit). Ces plans ou états de conscience, enfin, quand ils sont très intenses ou profonds, ne relèvent pas de la conscience vigile ordinaire et posent des questions générales d'ordre psychologique. D'où le titre et le deuxième thème de ce volume.

3 E. Franco rappelle sur ce point que les Yogasūtra de Patañjali faisaient une place importante aux réalisations et pouvoirs surnaturels (siddhi), cela du fait de l'influence bouddhique. Mais, note-t-il, si les disciples du Bouddha et du Jina (et leurs descendants) ont attribué à ces derniers ou à d'autres maîtres spirituels de tels pouvoirs, il n'est pas certain que ces maîtres y aient eux-mêmes prétendu. Les enseignements du Bouddha paraissent en effet avoir relevé surtout d'une approche rationnelle que la méditation n'avait qu'à confirmer tant par le raisonnement que par une vision intuitive. L. de la Vallée Poussin, auquel E. Franco se réfère ici, avait à cet égard cru naguère (en 1936-39) pouvoir distinguer, dans le bouddhisme ancien, deux approches de la connaissance salvatrice, l'une rationnelle, l'autre extatique ou «mystique», les pratiquants de cette dernière approche «touchant le nirvāna de leur corps» (conception qui sera celle des traditions tantriques). Mais ces deux approches sont-elles entièrement séparables? Il y a là une question que l'on verra apparaître dans plusieurs des contributions de ce volume; ce qui sera aussi le cas de la question, rappelée ici par l'auteur, du fondement, de la "vérité», des doctrines proposées. Mais quelle qu'ait pu être la valeur cognitive des expériences méditatives éventuellement «extrasensorielles» des diverses écoles, notamment bouddhiques, elles méritent une étude attentive. Cela aussi bien en raison de la place essentielle qu'elles occupent dans les traditions religieuses d'Asie du Sud et le bouddhisme que parce qu'elles peuvent apporter des lumières sur le fonctionnement de l'esprit humain et qu'elles sont parfois prises en compte dans certaines expériences d'ordre psychologique, ou utilisées en psychothérapie - ce point devant faire l'objet des contributions de la seconde partie du livre.

4 La première partie, "Yogic Perception in South Asian and Tibetan Traditions», rassemble dix contributions (pp.55-317), dont cinq concernent le bouddhisme. Elle s'ouvre par trois exposés qui réfutent ou mettent en doute la réalité ou la validité des expériences yogiques extrasensorielles. Celui, d'abord, de Lawrence McKrea, «"Just Like Us, Juste Like Now": The Tactical implications of the Mīmāṃsā Rejection of Yogic Perception", présente la position de la Mīmāmsāā, l'herméneutique du Veda, qui est de tous les systèmes brahmaniques/hindous le plus rigoureusement orthodoxe. Pour celleci, seule la Parole éternelle et autorévélée du Veda est valide et peut être la source d'une connaissance valable du dharma, la loi éternelle gouvernant le monde. Les éléments ou expériences surnaturelles que révèlerait le Bouddha, étant par nature 
imperceptibles à la généralité des êtres vivants, sont inutiles. En outre on ne peut pas faire confiance à la parole d'un Bouddha ou d'un yogin qui, comme tout autre être humain, peut être ignorant ou menteur; et s'il se trouvait que ce qu'il dit correspond à la réalité des choses, cela pourrait être découvert par tout un chacun avec des moyens ordinaires, sans recourir au yoga. On ne saurait être plus catégorique. On remarquera à cette occasion que les positions du plus agnostique bon sens peuvent se rencontrer au sein même du brahmanisme le plus orthodoxe. L'Inde compte aussi une ancienne tradition matérialiste: elle n'est pas, comme on le croit trop souvent, que le pays des yogins, du mysticisme et de la dévotion à mille divinités.

5 C'est à peu près le même thème, mais dans une optique toute différente, que traite ensuite John Taber en posant, dans son article, «Yoga and our Epistemic Predicament», la question, non de la possibilité d'une perception yogique (yogipratyakșa) extrasensorielle, mais celle de savoir si de telles expériences sont «vraies», si elles sont vraiment des moyens de connaissance. Il se réfère pour cela essentiellement au philosophe bouddhiste Ratnakīrti ( $\mathrm{x}^{\mathrm{e}} \mathrm{xI} \mathrm{e}^{\mathrm{e}}$ siècles), qui ne s'était toutefois pas posé exactement la même question - à laquelle il faut évidemment répondre que rien, sauf la croyance, ne peut affirmer la «vérité» de ces perceptions: tout ce qu'on peut faire, c'est en constater l'existence et l'importance dans leur contexte. Taber, lui, est moins sceptique. Il aimerait que l'on parvienne vis-à-vis de la connaissance yogique à une «revised theory of nature that can accomodate it», mais il reconnaît que, pour le moment, «it is not on the horizon».

6 La longue contribution d'Eli Franco, «Meditation and Metaphysics. On their Mutual Relationship in South Asian Buddhism», critique également certaines idées généralement reçues en mettant en doute la thèse, soutenue notamment naguère par Régamey et Conze et aujourd'hui par L. Schmithausen, selon laquelle ce sont des expériences yogiques méditatives et/ou extatiques qui ont été à l'origine d'aspects importants de la philosophie bouddhique - celle essentiellement du Grand Véhicule. E.Franco conteste en particulier la thèse de Schmithausen, que celui-ci, excellent spécialiste de ce domaine, tente d'établir de façon rigoureuse. C'est notamment la notion bouddhique d'anātman, d'inexistence du moi/soi, que Schmithausen croit fondée sur une expérience vécue du vide. Qu'une telle expérience soit possible, que l'on en tire la conviction qu'au fond il n'y a pas de soi, pourquoi pas? Mais on ne saurait pour autant affirmer, encore moins démontrer, qu'elle est à l'origine de la doctrine de l' anātman. Il est plus difficile encore de suivre Schmithausen quand il tente de trouver dans une expérience spirituelle yogique l'origine d'autres conceptions fondamentales du bouddhisme.

7 Les deux contributions suivantes examinent les conceptions des logiciens des écoles Madhyamaka et Yogacāra qui sont nécessairement fort subtiles et ingénieuses, car comment peut fonctionner la perception yogique quand on pose au départ qu'il n'y a rien à percevoir? Comment se libérer d'un monde sans réalité? Anne MacDonald, d'abord, «Knowing Nothing. Candrakīrti and Yogic Perception», traite de la perception yogique dans le Madhyamaka, école indienne importante du Grand Véhicule, qui a marqué le bouddhisme chinois et est encore vivante dans le bouddhisme tibétain. A.McDonald suit notamment le raisonnement de Candrakïrti (fl.600-650) pour qui la conscience d'un objet inexistant ne peut qu'être non existante; si le monde est irréel, la conscience que l'on en a l'est donc également. Situation inacceptable dont Candrakīti se tire en posant que la réalisation de la véritable nature des choses pourrait faire 
l'objet d'une gnose (jñāna) transcendant tout le divers, capable de percevoir l'inconcevable. L'étude brillante et ingénieuse de Vincent Eltschinger, «On the Career and the Cognition of yogins», qui traite de la pensée de Dharmakīrti, suit d'abord les étapes de la méditation et donc de la connaissance métaphysique d'un moine bouddhiste allant de son état premier d'être ordinaire, esclave du moi, à celui où il dépasse cet état, puis s'en délivre. V. Eltschinger envisage ensuite la dimension épistémologique de la connaissance que possède le yogin: par la paix et le discernenemt, il atteint un état de lucidité et de sagesse qui lui donne une connaissance intuitive (prajña) de la vraie nature (irréelle) de la réalité.

On reste dans le bouddhisme avec Dorji Wangchuk, «A Relativity Theory of the Purity and Validity of Perception in Indo-Tibetan Buddhism», qui examine ce qu'il nomme un aspect curieux de la théorie bouddhique de la connaissance: que des êtres de différentes espèces - humains, fantômes ou déités - perçoivent différemment les mêmes objets: une eau, pure pour les hommes, sera ainsi un flot impur pour les fantômes et du nectar pour les dieux. Ces êtres ont donc différentes approches cognitives: le cas est examiné avec soin par D.Wangchuk, comme s'il avait quelque réalité. C'est également du bouddhisme tibétain qu'il s'agit dans l'article d'Orna Almogi, "The Materiality and Immanence of Gnosis in Some rNying-ma Tantric Sources», traitant de ce qu'elle nomme «the meta-physiology of the gnosis», c'est-à-dire d'une sapience vécue corporellement - essentiellement dans le «cœur» en tant que point nodal (cakra) du corps imaginal yogique dont la structure de centres et de conduits est imaginée comme présente («intraposed») dans le corps physique de l'adepte. Le bouddhisme tibétain reprend là en les adaptant les conceptions du hathayoga tantrique - on sait, de fait, que le Canon bouddhique tibétain est très largement constitué de traductions ou transpositions de tantras śivaïtes.

On en vient d'ailleurs au domaine hindou dans les trois communications suivantes. André Philippe Maas, d'abord, examine «The So-Called Yoga of Suppression in the Pātañjala Yogaśāstra ", texte dont A.P. Maas a récemment procuré une excellente édition. Comme le fait observer Eli Franco dans son introduction, «yoga of suppression» n'est pas une expression satisfaisante pour rendre le sanskrit yogaś cittavrttinirodhah (que Maas traduit assez maladroitement par «the shutdown of the processes of mental capacity»). Mais cette première étape du yoga, celle d'un arrêt ou d'une mise à l'écart des mouvements mentaux pour que ne subsiste qu'une conscience "pure» où apparaitra le divin, est importante et l'étude qui en est faite ici n'est pas inutile.

10 Le yoga étant, outre une vision de la réalité (un darśana), une technique somatopsychique, son rapport à la dévotion, la bhakti, où la grâce divine joue un rôle essentiel, peut poser un problème, car faire effort pour aller vers Dieu est un mouvement contraire à celui de la grâce divine qu'il faut et qu'il suffit de recevoir: Rāmānuja, le fondateur du Viśișțadvaita Vedānta, en était conscient. Mais on peut aussi penser que la divinité agit comme un yogin et dès lors qu'une approche et une connaissance d'ordre yogique de la divinité sont possibles et valables, position qui serait celle du viśiștādvaitin Meghanādārisūri (XIII ${ }^{\mathrm{e}}$ siècle) étudiée par Markus Schmükker.

11 Marion Rastelli, enfin («Perceiving God and Becoming Like Him: Yogic Perception and its Implications in the Viśnuitic Tradition of Pāñcarātra»), traite également de conceptions vishnouites, mais tirées de textes du Pāñcarātra, qui est une tradition tantrique, ancienne et toujours vivante. La méditation yogique y tend à l'identification 
(mais non à la fusion totale) de l'adepte avec la déité, à laquelle il peut parvenir par un processus mental, mais aussi et surtout au moyen d'un mantra dont il évoquera et suivra mentalement, en s'y identifiant, la dissolution dans l'absolu; ou encore, plus rituellement, par une dévotion identificatrice avec une des formes, mentalement évoquée (et donc rendue présente), de la divinité: autant d'aspects de l'efficacité cognitive et salvatrice d'une pratique yogique où, en fait, les créations imaginaires et les visualisations jouent un rôle essentiel.

Bouddhiques ou hindoues, les pratiques envisagées dans les contributions de cette première partie visent toutes à créer des perceptions paranormales ou extrasensorielles. La conscience ne s'y applique plus à des réalités normalement perceptibles, non plus qu'à des spéculations abstraites, à une pensée discursive. "Vidée», immobilisée, «transcendée», mystiquement unie à la divinité, la conscience du pratiquant n'est plus dans une condition vigile «normale»: elle est dans ce qu'on est convenu d'appeler en anglais - du moins depuis l'ouvrage en un temps classique de Charles T.Tart - un «altered state of consciousness» (ASC) - état dont entend traiter sur un plan général la seconde partie du livre: «Meditation and Altered States of Consciousness from an Interdisciplinary Perspective» (pp.321-479).

Le sujet mérite en effet d'être examiné. Mais il aurait dû l'être dans une étude d'ensemble qui aurait tâché de cerner le problème des «ASC» en liaison avec les cas présentés dans la première partie - encore eût-il fallu, pour cela, un psychologuepsychiatre au fait des récentes données des neurosciences, en même temps qu'orientaliste, ce qui ne doit pas être facile à trouver. Cette partie comporte sept articles sur des thèmes divers. Le premier, de Karl Baier, présentant rapidement «Meditation and Contemplation in High to Late Europe», ne pouvait guère que rappeler quelques faits bien connus. L'étude de Diana Riboli sur des shamans au Népal et en Malaisie et celle de Dagmar Eigner sur des shamans et des mediums du Népal central ne sont pas inintéressantes, mais sans apporter rien de nouveau sur les sujets présentés. "Altered States of Consciousness as Structural Variations of the Cognitive Systems», de Shulamith Kreitler, propose une théorie interprétative abstraite et, me semble-t-il, peu compréhensible. Cette seconde partie est décevante, mais peut-être ne pouvait-elle que l'être. On ne saurait en faire reproche à Eli Franco, qui était d'ailleurs conscient (voir p. 50) des insuffisances de cette portion finale du volume qu'il a dirigé, dont la première et plus longue section (317 pages) - l'introduction et les contributions orientalistes est d'une excellente qualité, apportant sur certains sujets des vues nouvelles, des éclairages intéressants. L'ouvrage mérite à cet égard tout à fait d'être lu ou consulté. 